\title{
Contralateral posterior interhemispheric approach to deep medial parietooccipital vascular malformations: surgical technique and results
}

\author{
Jan-Karl Burkhardt, MD, Ethan A. Winkler, MD, PhD, and Michael T. Lawton, MD \\ Department of Neurological Surgery, University of California, San Francisco, California \\ OBJECTIVE Deep medial parietooccipital arteriovenous malformations (AVMs) and cerebral cavernous malforma- \\ tions (CCMs) are traditionally resected through an ipsilateral posterior interhemispheric approach (IPIA), which creates \\ a deep, perpendicular perspective with limited access to the lateral margins of the lesion. The contralateral posterior \\ interhemispheric approach (CPIA) flips the positioning, with the midline positioned horizontally for retraction due to grav- \\ ity, but with the AVM on the upper side and the approach from the contralateral, lower side. The aim of this paper was to \\ analyze whether the perpendicular angle of attack that is used in IPIA would convert to a parallel angle of attack with the \\ CPIA, with less retraction, improved working angles, and no significant increase in risk.
}

METHODS A retrospective review of pre- and postoperative clinical and radiographic data was performed in 8 patients who underwent a CPIA.

RESULTS Three AVMs and 5 CCMs were resected using the CPIA, with an average nidus size of $2.3 \mathrm{~cm}$ and CCM diameter of $1.7 \mathrm{~cm}$. All lesions were resected completely, as confirmed on postoperative catheter angiography or MRI. All patients had good neurological outcomes, with either stable or improved modified Rankin Scale scores at last follow-up.

CONCLUSIONS The CPIA is a safe alternative approach to the IPIA for deep medial parietooccipital vascular malformations that extend $2 \mathrm{~cm}$ or more off the midline. Contralaterality and retraction due to gravity optimize the interhemispheric corridor, the surgical trajectory to the lesion, and the visualization of the lateral margin, without resection or retraction of adjacent normal cortex. Although the falx is a physical barrier to accessing the lesion, it stabilizes the ipsilateral hemisphere while gravity delivers the dissected lesion through the transfalcine window. Patient positioning, CSF drainage, venous preservation, and meticulous dissection of the deep margins are critical to the safety of this approach. https://thejns.org/doi/abs/10.3171/2017.2.JNS162750

KEY WORDS arteriovenous malformation; cerebral cavernous malformation; medial parietooccipital lobe; cingulate cortex; corpus callosum; contralateral posterior interhemispheric approach; ipsilateral posterior interhemispheric approach; vascular disorders; surgical technique

$\mathrm{V}$ ASCULAR lesions located in the medial parietooccipital lobes are traditionally resected using an ipsilateral posterior interhemispheric approach (IPIA). ${ }^{8}$ The IPIA is a versatile approach that we have used for a variety of vascular pathology in the posterior midline, including resection of arteriovenous malformations (AVMs), galenic dural arteriovenous fistulas (DAVFs), cerebral cavernous malformations (CCMs), and distal posterior cerebral artery (PCA) aneurysms. ${ }^{2}$ In a previous review of 46 patients in whom the IPIA was performed in either the prone or lateral position, we demonstrated the benefits of the lateral position, with gravity retracting the occipital lobe, enhancing operative exposure, and reducing morbidity. ${ }^{2}$ Since that report in 2006, gravity-assisted IPIA has been used to expose medial parietooccipital vascular pathology with a torcular craniotomy that completely exposes the midline, orients it horizontally, and liberates the occipital pole to descend with retraction due to gravity. However, this approach creates a deep, perpendicular perspective with limited access to the lateral margins of the lesion. For example, an AVM is dissected up from the sagging occipital lobe, drawing the AVM medially into the interhemispheric fissure with restricted access to the lateral margins that often receive supply from challenging

ABBREVIATIONS $\mathrm{AVM}=$ arteriovenous malformation; $\mathrm{CAIA}=$ contralateral anterior interhemispheric approach; $\mathrm{CCM}=$ cerebral cavernous malformation; $\mathrm{CPIA}=$ contralateral posterior interhemispheric approach; DAVF = dural arteriovenous fistula; DVA = developmental venous anomaly; IAIA = ipsilateral anterior interhemispheric approach; IPIA = ipsilateral posterior interhemispheric approach; ISS = inferior sagittal sinus; IVH = intraventricular hemorrhage; $m R S=$ modified Rankin Scale; PCA = posterior cerebral artery; SSS = superior sagittal sinus.

SUBMITTED October 31, 2016. ACCEPTED February 2, 2017.

INCLUDE WHEN CITING Published online July 21, 2017; DOI: 10.3171/2017.2.JNS162750. 
atrial arteries or perforators traveling through deep white matter. A similar problem has been encountered with the ipsilateral anterior interhemispheric approach (IAIA) for medial frontal AVMs, and our solution to that problem in the frontal lobe was to approach the pathology from the contralateral side.?

The contralateral anterior interhemispheric approach (CAIA) flips the positioning, still with the midline positioned horizontally for retraction due to gravity, but with the AVM on the upper side and the approach from the contralateral, lower side (i.e., the side closer to the floor). This geometry provides the same exposure of the medial hemisphere and the AVM's midline anatomy, but, with circumferential dissection around the nidus, gravity delivers the nidus into the interhemispheric fissure and improves access to treacherous lateral and deep margins. In addition, the crossing-midline trajectory requires less retraction and/or resection of adjacent cortex than the IAIA.

An analogous solution can be applied in the posterior interhemispheric fissure with a contralateral posterior interhemispheric approach (CPIA) (Fig. 1). The CPIA also flips the positioning, with the midline positioned horizontally for retraction due to gravity, but the AVM on the upper side and the approach from the contralateral, lower side. The CPIA involves the unaffected contralateral occipital lobe, and the falx is a barrier to access. In addition, the reach to the lateral border is longer, and intraoperative bleeding would be more difficult to manage from the contralateral side. However, favorable experience with the CAIA encouraged us to use the CPIA. Therefore, the CPIA was performed in 8 patients with the hypothesis that the perpendicular angle of attack associated with the IPIA would convert to a more favorable parallel angle of attack with the CPIA, with less retraction, improved working angles, and no significant increase in risk.

\section{Methods \\ Patients}

The institutional review board approved this study. A retrospective review of patients treated with the CPIA performed by the senior author between January 1998 and September 2016 identified 8 patients who harbored AVMs or CCMs. The indications for this technique were based on the preoperative images demonstrating a lesion extending approximately $2 \mathrm{~cm}$ or more off the posterior midline. Clinical data (e.g., age, sex, and presenting symptoms), radiographic data (e.g., AVM laterality, feeding arteries, draining veins, and involved cortical structures), and AVM grades (Spetzler-Martin grade, ${ }^{11}$ Lawton-Young supplementary grade, ${ }^{10}$ and supplemented Spetzler-Martin grade $^{10}$ ) were collected. Patient outcomes were measured using the modified Rankin Scale (mRS) scores assessed by an independent research nurse.

\section{Surgical Technique for the CPIA}

The patient is positioned laterally with the ipsilateral hemisphere harboring the vascular lesion positioned on the upper side to allow gravity to retract the contralateral hemisphere (Fig. 1). The head is turned nose down about $60^{\circ}$ toward the floor, with slight neck flexion (Fig. 1A, right).
With this position, and facing the field from behind the patient, the posterior third of the superior sagittal sinus (SSS) is horizontal, the occipital pole falls away from the falx, and the interhemispheric fissure is widened by gravity retraction. The head is fixed in a Mayfield head holder. Neuronavigation is registered to precisely plan the approach trajectory and avoid vascular structures when making the transfalcine incisions. A lumbar drain or ventriculostomy is needed to drain cerebrospinal fluid and relax the brain, especially when a smaller craniotomy is used instead of a torcular craniotomy. A semicircular scalp incision exposes the anterior margin of the surgical corridor, crosses the midline, and curves inferiorly along the SSS, which allows the scalp flap to mobilize inferolaterally and also convert to a larger torcular exposure if necessary. A small biparietal or bioccipital craniotomy is performed that places most of the craniotomy on the contralateral side but crosses the SSS and exposes some of the ipsilateral side. The dura is opened in a semicircular flap based along the SSS and tacked up medially. Releasing arachnoidal adhesions between the dura and the paramedian hemisphere opens the contralateral interhemispheric fissure (Fig. 1B, right). Bridging lateral veins course anteriorly, and the interhemispheric approach is centered behind them. Subarachnoid dissection deepens down the falcotentorial junction to the incisura to open the callosal and quadrigeminal cisterns, drain CSF, and slacken the brain, which further drops the occipital pole and widens the surgical corridor. Next, a transfalcine window is made between the SSS and the inferior sagittal sinus (ISS) and straight sinus (Fig. 1C and $\mathrm{D}$, right). With AVMs, feeding arteries and draining veins may adhere to the falx, and these incisions are performed layer by layer with counter-traction away from the nidus. Subarachnoid dissection crosses through the falx and into the ipsilateral interhemispheric fissure to expose the lesion. With AVMs, the medial part of the nidus is de-arterialized and then circumferential dissection exposes the lateral margin. The nidus mobilizes into the interhemispheric fissure with gravity, and the falx holds the ipsilateral hemisphere in position (Fig. 1E, right). Finally, the draining veins are cauterized and divided after they darken, allowing the nidus to be removed en bloc. This approach increases visualization of the lateral border, which is typically the most difficult one to see (Fig. 1F, right). With CCMs, the lesion is circumferentially dissected and removed en bloc, with associated developmental venous anomalies (DVAs) preserved. Unlike the IPIA, no mechanical brain retraction is needed in the contralateral approach.

\section{Results}

During an 18-year period between 1998 and 2016, the senior author microsurgically treated 736 brain AVMs and 670 CCMs. The CPIA was only adopted recently and used in 8 patients, of whom 3 had AVMs and 5 had CCMs (Tables 1 and 2). The median age was 56 years (range 16-68 years); 3 patients were female and 4 were male. Two AVM patients and $1 \mathrm{CCM}$ patient presented with hemorrhage. All patients were right-handed, and all had their lesion in the right hemisphere. The average AVM diameter was 2.3 $\mathrm{cm}$. All 3 patients had Spetzler-Martin Grade II AVMs, 


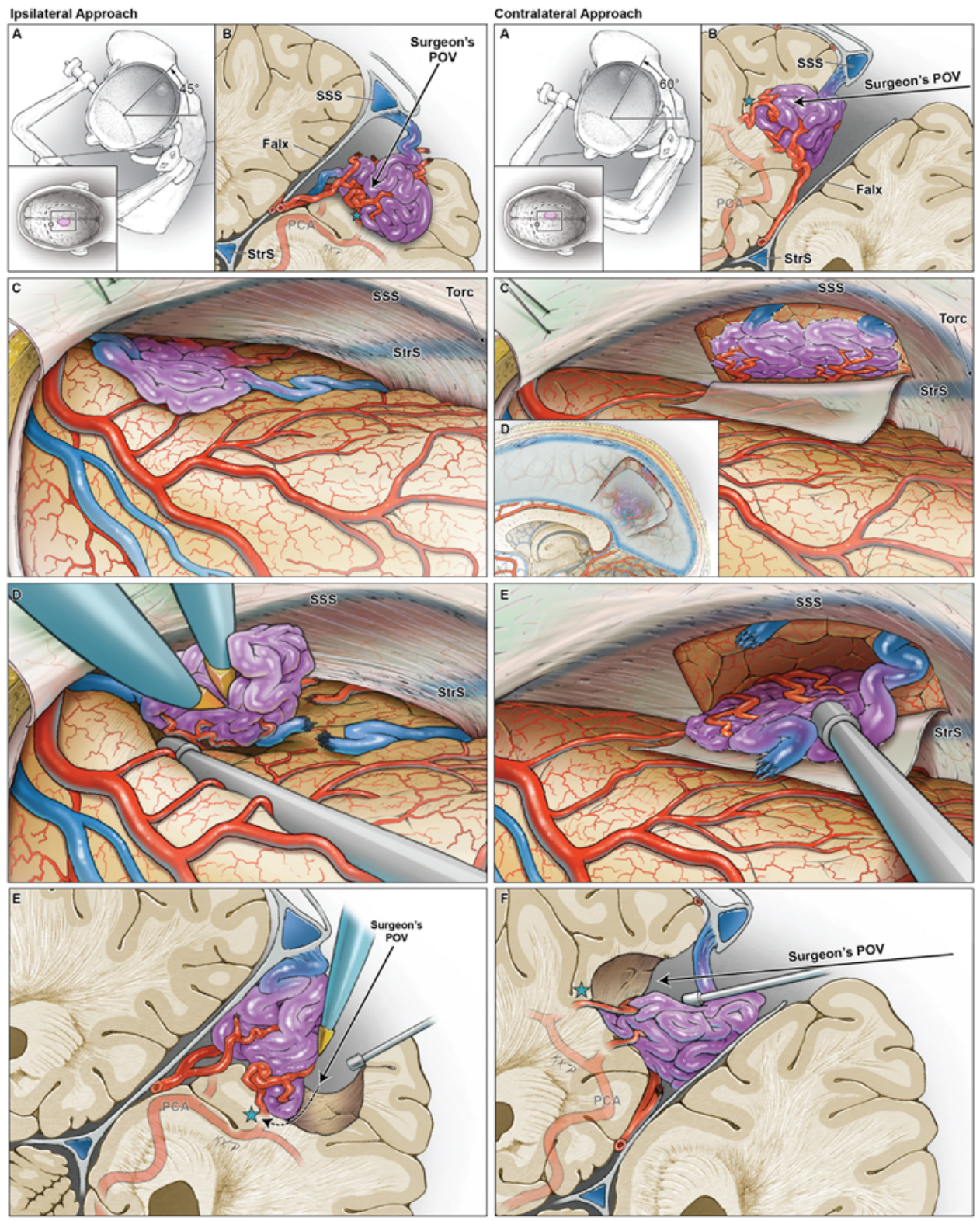

FIG. 1. Overview of surgical technique with the IPIA (left panels) and CPIA (right pane/s) for AVM resection. Left. A: For the IPIA, the head is positioned with the midline horizontal. In the inset, the purple area represents the lesion; the dashed line, the scalp incision; the circle, the bur hole; and the solid line, the bifrontal craniotomy. The left AVM is on the down side, and the head is angled $45^{\circ}$ upward from horizontal. B: In the coronal cross-sectional view, the neurosurgeon's point of view (POV) is almost vertically downward toward the lower medial hemisphere, which makes the lateral margin (star) inaccessible without additional retraction or tissue resection along the arrow. C-E: Surgeon's view (C) demonstrating excellent access to the medial AVM margin and feeders from the left PCA (D), but the nidus must be mobilized into the interhemispheric fissure against gravity and the frontal lobe must be retracted or some tissue resected to circumdissect the lateral margin (E). The dashed arrow indicates the coronal cross-sectional view. Right. A: For the CPIA, the head is positioned with the midline horizontal. In the inset, the dashed line represents the scalp incision; the circle, the bur hole; and the solid line, the bifrontal craniotomy. The right AVM is on the up side, and the head is angled $60^{\circ}$ upward from horizontal. B: In the coronal cross-sectional view, the neurosurgeon's point of view is almost horizontal over the lower medial hemisphere and across the interhemispheric fissure, which makes the lateral margin (star) accessible without additional retraction or tissue resection. C and D: Surgeon's view demonstrating excellent access to the medial AVM margin and feeders from the right PCA (C), after incising and opening the falx between the SSS and the straight sinus $(S t r S ; D)$. $E$ and $F$ : The nidus falls into the interhemispheric fissure with gravity (E), providing a direct view of the lateral margin $(F)$, thereby facilitating circumdissection of the AVM's most difficult margin (dashed arrow, coronal cross-sectional view). Torc = torcular. Copyright Kenneth Xavier Probst. Published with permission.

whereas Lawton-Young supplementary grades were more variable. The average CCM size was $1.7 \mathrm{~cm}$, and all patients had a CCM-associated DVA.
No surgical complications were observed. All AVMs were resected completely, as confirmed by postoperative catheter angiography, and all CCMs were resected com- 
TABLE 1. Characteristics of patients who underwent the CPIA for the resection of AVMs

\begin{tabular}{|c|c|c|c|c|c|c|c|c|c|c|c|c|c|}
\hline $\begin{array}{l}\text { Case } \\
\text { No. }\end{array}$ & $\begin{array}{c}\text { Age } \\
\text { (yrs), } \\
\text { Sex }\end{array}$ & $\begin{array}{l}\text { Presenting } \\
\text { Symptoms }\end{array}$ & $\begin{array}{l}\text { AVM } \\
\text { Side }\end{array}$ & $\begin{array}{l}\text { Nidus } \\
\text { Size } \\
(\mathrm{cm})\end{array}$ & $\begin{array}{l}\text { SM } \\
\text { Grade }\end{array}$ & $\begin{array}{l}\text { Suppl } \\
\text { Grade* }\end{array}$ & $\begin{array}{l}\text { Feeding } \\
\text { Arteries }\end{array}$ & Draining Vein(s) & $\begin{array}{l}\text { Structure } \\
\text { Involved }\end{array}$ & $\begin{array}{l}\text { Preop } \\
\text { Embo }\end{array}$ & $\begin{array}{c}\text { Preop } \\
\text { mRS } \\
\text { Score }\end{array}$ & $\begin{array}{c}\text { Postop } \\
\text { mRS } \\
\text { Score }\end{array}$ & $\begin{array}{r}\mathrm{EBL} \\
(\mathrm{ml})\end{array}$ \\
\hline 1 & $56, \mathrm{M}$ & $\mathrm{HA}, \mathrm{IVH}$ & Rt & 1.9 & ॥ & 3 & $\begin{array}{l}\text { Lat PChA, SplenA, } \\
\text { PcaA, CmaA }\end{array}$ & PcaV, VoG & Splenium & 1 & 1 & 0 & 500 \\
\hline 2 & $68, M$ & $\mathrm{HA}$ & $\mathrm{Rt}$ & 2.5 & II & 4 & PcaA & PCV, MPV, SSS & Cing cortex, PCL & 1 & 0 & 0 & 300 \\
\hline 3 & $16, M$ & $\mathrm{ICH}, \mathrm{IVH}$ & Rt & 2.5 & II & 1 & $\begin{array}{c}\text { Lat PChA, medial } \\
\text { PChA, PcaA }\end{array}$ & PcaV, VoG & Splenium & 1 & 1 & 1 & 500 \\
\hline
\end{tabular}

Cing = cingulate $\mathrm{CmaA}=$ callosomarginal artery; $\mathrm{EBL}=$ estimated blood loss; embo = embolization; $\mathrm{HA}=$ headache; $\mathrm{ICH}=$ intracranial hemorrhage; $\mathrm{MPV}=$ medial parietal vein; $\mathrm{PChA}=$ posterior choroidal artery; $\mathrm{PCV}=$ paracentral vein, $\mathrm{PcaA}=$ pericallosal artery; $\mathrm{PcaV}=$ pericallosal vein; $\mathrm{PCL}=$ paracentral lobule; $\mathrm{SplenA}=$ splenial artery; SM = Spetzler-Martin; Suppl = Supplementary; VoG = vein of Galen .

* Lawton-Young supplementary grade.

pletely, as confirmed by MRI. One patient with a CCM had a small remnant that was seen on postoperative MRI and required reoperation through the same approach 4 days after the first surgery. All patients had good neurological outcomes (mRS Score 0 or 1), with either stable or improved mRS scores at last follow-up. The median duration of follow-up was 52 days (range 19-112 days).

\section{Illustrative Cases \\ Case 1}

A right-handed 56-year-old man presented with worsening occipital headache for 4 weeks and nausea/vomiting for 3 days. A right intraventricular hemorrhage (IVH) from a ruptured medial parietal periventricular AVM was diagnosed (Fig. 2A). Preoperative angiography demonstrated a compact AVM nidus adjacent to the right splenium with the arterial supply from branches of the right lateral posterior choroidal artery and right splenial artery (Fig. 2B and C). Additional feeders derived from the distal right pericallosal and callosomarginal arteries (Fig. 2B and C). A 3-mm inferiorly directed intranidal aneurysm was seen at the deep portion of the AVM nidus. Drainage was deep to the vein of Galen without venous outflow stenosis. The right lateral posterior choroidal artery, right splenial artery, and the intranidal aneurysm were embolized preoperatively. The AVM was microsurgically resected through a CPIA with the left hemisphere in the lower position and the right-sided AVM on the upper side (Fig. 2D). The CPIA was performed through a torcular craniotomy. After opening the contralateral interhemispheric fissure and draining CSF from the quadrigeminal cistern to relax the brain, the transfalcine window was opened to the ipsilateral interhemispheric fissure (Fig. 2E). The arterialized draining vein was seen emptying into the vein of Galen. Feeders to the AVM were followed along their course from the callosomarginal and pericallosal vessels anteriorly and the splenial artery posteriorly and then coagulated and divided. Circumdissection into the cingulate gyrus defined the superior margin of the AVM (Fig. 2F), and the atrium of the lateral ventricle was entered. The crossing trajectory of the CPIA provided a direct view of the lateral AVM margin, and gravity delivered the nidus into the interhemispheric fissure. The nidus was removed en bloc (Fig. 2G and H). Postoperative angiography and MRI showed no residual AVM nidus (Fig. 2I and J). The patient's postoperative course was notable for transient decreased vision in bilateral visual fields, which resolved completely within the 1st week after surgery. The patient had no neurological deficits at the 6-week follow-up.

\section{Case 6}

A right-handed 60-year-old woman presented after 3 focal seizures while on a regimen of appropriate anticonvulsant therapy, and MRI showing a CCM in the right precuneus with an anterior inferior DVA draining ependymally (Fig. 3A). Lesionectomy was recommended for seizure control and protection against future hemorrhage. Rather than a torcular craniotomy, a smaller biparietal craniotomy was performed; a lumbar drain was placed to relax the brain. The CCM was microsurgically resected through a CPIA with the left hemisphere in the lower position and the right-sided CCM on the upper side (Fig. 3B-F). Detaching adhesions between the parietooccipital lobes and the falx opened the ipsilateral interhemispheric

TABLE 2. Characteristics of patients who underwent the CPIA for the resection of CCMs

\begin{tabular}{|c|c|c|c|c|c|c|c|c|c|}
\hline $\begin{array}{l}\text { Case } \\
\text { No. }\end{array}$ & $\begin{array}{c}\text { Age (yrs), } \\
\text { Sex }\end{array}$ & Presenting Symptoms & $\begin{array}{l}\text { CCM } \\
\text { Side }\end{array}$ & $\begin{array}{c}\text { CCM Size } \\
(\mathrm{cm})\end{array}$ & $\begin{array}{c}\text { DVA } \\
\text { Present }\end{array}$ & Structure Involved & $\begin{array}{c}\text { Preop } \\
\text { mRS }\end{array}$ & $\begin{array}{c}\text { Postop } \\
\text { mRS }\end{array}$ & $\begin{array}{l}\text { EBL } \\
(\mathrm{ml})\end{array}$ \\
\hline 4 & $61, F$ & HA, progressive hemisensory loss & $\mathrm{Rt}$ & 2.4 & Yes & Splenium, posterolat thal & 1 & 0 & 500 \\
\hline 5 & $32, \mathrm{M}$ & $\mathrm{HA}, \mathrm{ICH}$ & Rt & 1.8 & Yes & Splenium & 1 & 0 & 500 \\
\hline 6 & $60, \mathrm{~F}$ & Focal seizures & Rt & 1.6 & Yes & Precun cortex & 1 & 0 & 100 \\
\hline 7 & $32, \mathrm{~F}$ & Focal seizure & Rt & 2.1 & Yes & Precun cortex & 1 & 0 & 300 \\
\hline 8 & $36, \mathrm{M}$ & Focal seizures & $\mathrm{Lt}$ & 0.8 & Yes & Precun cortex & 1 & 1 & 100 \\
\hline
\end{tabular}

DVA = developmental venous anomaly; precun = precuneus; thal = thalamus. 

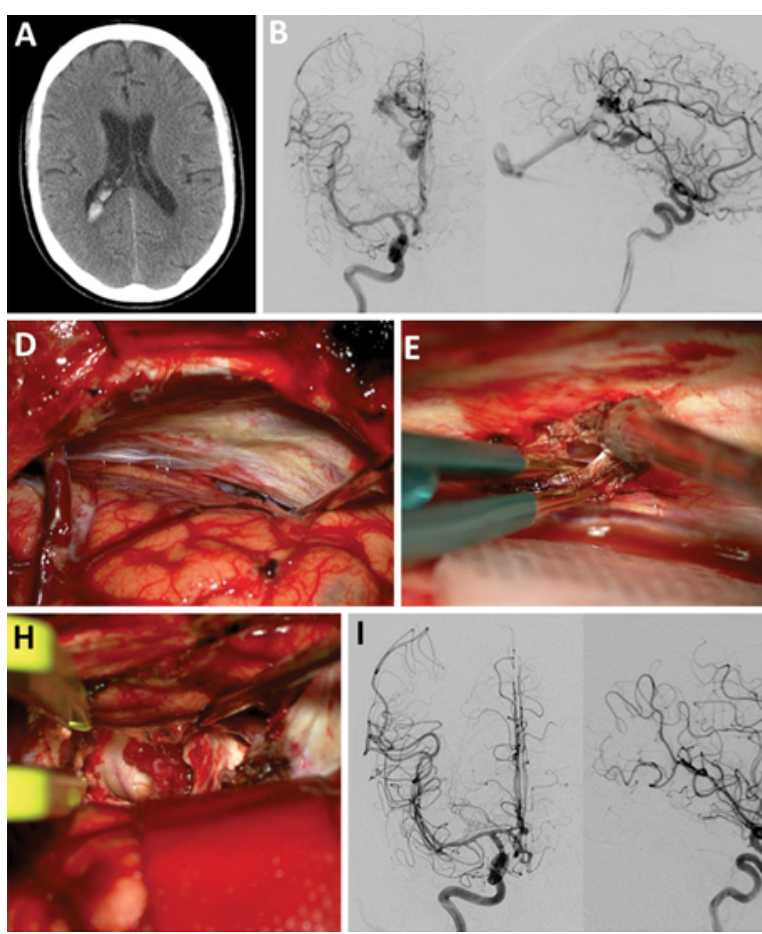

FIG. 2. Case 1. A: Preoperative axial CT demonstrating a right IVH from a ruptured medial parietal periventricular AVM. B and C: Preoperative anteroposterior and lateral digital subtraction angiograms as well as a coronal T2-weighted MR image demonstrate a compact AVM nidus adjacent to the right splenium with the arterial supply from branches of the right lateral posterior choroidal artery and right splenial artery. Additional feeders are derived from the distal right pericallosal and callosomarginal arteries. D-H: Intraoperative photographs show the contralateral view to the falx via the CPIA (D), opening of the falx (E), and resection of the AVM (F); the AVM was removed en bloc $(G)$ with no AVM remnant $(H)$. I and J: Postoperative anteroposterior and lateral digital subtraction angiograms as well as a coronal T1-weighted MR image with contrast showing no residual arteriovenous shunting and complete resection of the AVM.

fissure. The quadrigeminal cistern was opened for additional CSF release, and an incision was made in the falx to enter the ipsilateral interhemispheric fissure. The CCM was identified just below the cortical surface, and it was removed en bloc after circumferential dissection, with preservation of the associated DVA. Postoperatively, the patient had no new neurological deficits, and postoperative MRI showed no residual CCM (Fig. 3G). The patient has had no further seizures.

\section{Discussion}

Contralateral approaches through the interhemispheric fissure are designed to access lesions located laterally in or adjacent to the lateral ventricle, without retracting or resecting normal brain tissue en route to the lesion. Contralateral approaches create a crossing trajectory that begins in the interhemispheric fissure contralateral to the lesion but ends in the medial hemisphere or the ventricle ipsilateral to the lesion. A variety of contralateral approaches have been introduced as this concept has gained acceptance over time. The contralateral transcallosal approach-with the head positioned on the midline oriented horizontally and the lesion on the upper side to allow gravity to retract the lower hemisphere and open the interhemispheric fissure-accesses small lesions in the ventricular wall, caudate head, and thalamus. ${ }^{9}$ The contralateral transcingulate approach is a variation of the contralateral transcallosal approach, but it does not deepen through the corpus callosum or enter the ventricle; rather, it crosses with a shallower trajectory to lesions in the cingulate gyrus and deep white matter of the medial frontal lobe. ${ }^{3}$ This approach targets small lesions located between the pericallosal and callosomarginal arteries, whereas the transcallosal approach dives beneath the pericallosal artery. The CAIA is an extension of the contralateral transcingulate approach, accessing larger lesions not only in the cingulate gyrus but also in the medial frontal lobe through a window in the falx for more exposure.

In this current report, a favorable experience with the CPIA for vascular lesions justifies its addition to the menu of contralateral interhemispheric approaches. Similar advantages in exposure and access result from retraction due to gravity and a crossing trajectory with the CPIA to lesions in the medial parietal and occipital lobes, but this approach has not been widely used with AVMs and CCMs for several reasons. First, an approach from the contralateral side exposes both hemispheres to surgical manipulation and risk of injury. Second, the falx is a midline barrier that conceals AVM anatomy and must be incised to access the lesion. Third, the surgical corridor is deepened with a crossing trajectory. And finally, intraoperative AVM hemorrhages at increased depths and in a hemisphere that is only minimally exposed by the craniotomy might be more dangerous and difficult to control.

In an earlier publication in 2006, we found that grav- 

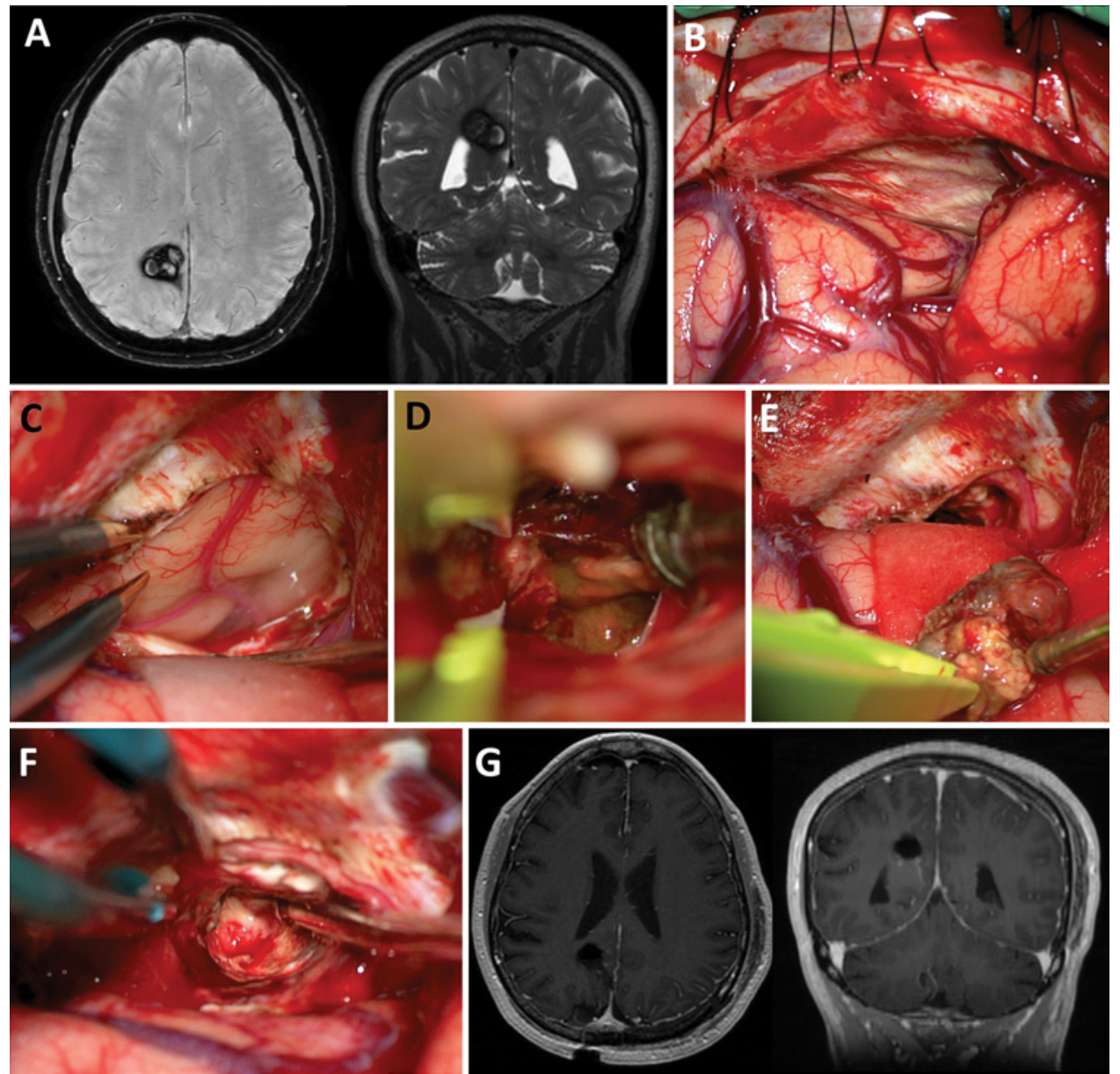

FIG. 3. Case 7. A: Preoperative axial T2*-weighted and coronal T2-weighted MR images demonstrating a CCM in the right precuneus. B and C: Intraoperative photographs showing the CPIA by opening the contralateral interhemispheric fissure (B) and opening of the falcine window (C). D-F: The CCM was removed completely ( $D$ and $E$ ) and the CCM resection cavity showed no remnant $(F)$. G: Postoperative axial and coronal T1-weighted MR images with contrast, showing no residual CCM with intact developmental venous anomalies.

ity retraction greatly enhanced the exposure of the IPIA. ${ }^{2}$ That publication also included our first experience with the CPIA, but this case was performed with the patient prone without gravity retraction, and we abandoned it for many years. Goel ${ }^{6}$ was one of the first to report the use of the CPIA in 4 patients, but the details about patient position were not included. Ferroli et al. reported a case of a subcortical ependymoma that was resected via a gravityassisted CPIA, ${ }^{4}$ with the patient supine and the shoulder bolstered, the midline of the head turned parallel to the floor, and the head tilted upward $45^{\circ}$, as with a contralateral transcallosal approach. This position allowed access to the middle third of the interhemispheric fissure, but it is not ideal for the posterior third. Bohnstedt et al., in an experience with a transprecuneus approach to periatrial tumors and AVMs, used a three-quarter prone position that places the ipsilateral hemisphere up and aligns the axis of the SSS at a $45^{\circ}$ angle with the floor. ${ }^{1}$ Our patients were positioned similarly, except that the axis of the midline is turned more downward $\left(60^{\circ}\right)$. By facing the field from behind the patient, the posterior third of the SSS is horizontal, and the occipital pole falls away from the falx with gravity to widen the interhemispheric fissure. Early drainage of CSF from the quadrigeminal and ambient cisterns widens the fissure further.

With the CAIA to frontal lesions, the falx is narrow and may not even conceal the pathology on the opposite side. In contrast with the CPIA, the falx is broad and anchored to the falcotentorial junction with the ISS, galenic complex, and straight sinus coursing through its base. A generous transfalcine window must be opened to reach the pathology on the opposite side. We prefer a trap door incision in the falx with a horizontal incision just below the SSS and 2 vertical incisions down to the ISS anteriorly and straight sinus posteriorly. The ISS can be occluded and transected without injury, freeing the anterior base of the falcine flap, but the straight sinus must be preserved in the posterior base. The trap door mobilizes contralaterally to protect that medial hemispheric surface. In their report, Bohnstedt et al. described a T-shaped incision in the falx that opens a triangular transfalcine window. ${ }^{1}$ The transfalcine window creates an entrance to the opposite side, and the remaining falx holds the ipsilateral hemisphere in place while allowing gravity to deliver the AVM or CCM 
into the interhemispheric fissure as the lesion is circumferentially dissected. Therefore, gravity both widens the posterior interhemispheric fissure and opens the plane of dissection around the lateral margin of the lesion for a direct view of the most treacherous part of the dissection. These double duties of gravity retraction with the CPIA are not realized with an ipsilateral approach. Parietooccipital vascular malformations with a lateral margin extending more than $2 \mathrm{~cm}$ from the midline are difficult to remove safely through the IPIA because exposure of this lateral margin requires retraction or resection of the medial aspect of the cuneus and lingual gyrus for lesions in the occipital lobe, and the postcentral gyrus and superior parietal gyrus for lesions in the parietal lobe. Retraction or brain resection in this territory can damage primary sensory, supplementary sensory, and/or primary visual cortex. In addition, the superficial draining veins of medial AVMs ascend in the interhemispheric fissure to the SSS and create obstacles in the surgical corridor of the IPIA. In contrast, these veins lie above the surgical corridor of the CPIA and are protected by the falx.

Although this study is limited by the small number of patients, selection biases, and retrospective nature, it demonstrates important advantages of contralaterality for vascular lesions in the deep medial parietooccipital region. We did not observe any long-term morbidity due to hemorrhage or stroke in either the contra- or ipsilateral hemisphere. One patient (Case 1) developed postoperative bilateral visual loss, but this deficit resolved completely 1 week after surgery. This transient deficit was caused by retraction of the visual cortex during surgery, even without fixed retractor blades, and was observed in our previous reports on the IPIA. ${ }^{2}$ Neuronavigation was useful in identifying bridging veins preoperatively to plan the craniotomy flap and preserve them in all cases. ${ }^{5}$ The transfalcine window was created without causing complications to the deep venous system or straight sinus. Although this study confirms the safety of the CPIA for posterior midline AVMs and CCMs extending laterally, some ruptured AVMs may be better accessed ipsilaterally, where a large hematoma may offer a more direct approach with a shorter working distance transcortically through the evacuated clot. AVMs associated with small hematomas or IVH may still benefit from the CPIA, as shown in Cases 1 and 3. Small AVMs or CCMs located in the midline without lateral extension are best reached through an IPIA.

\section{Conclusions}

This report describes our touches to the CPIA, which include our patient positioning, shape of the falcine window, and patient inclusion criteria. Despite previous knowledge of this approach, we were hesitant to perform it, and it took a long time for us to adopt it. We suspect that others share these hesitations and have not taken these steps, and we offer this experience to help others take this step. The CPIA is an alternative approach to the IPIA for deep, medial parietooccipital vascular malformations that extend $2 \mathrm{~cm}$ or more off the midline. Contralaterality and gravity retraction optimize the interhemispheric corridor, the surgical trajectory to the lesion, and the visualization of the lateral margin, without resection or retraction of adjacent normal cortex. Although the falx is a physical barrier to lesion access, it stabilizes the ipsilateral hemisphere while gravity delivers the dissected lesion through the transfalcine window. Patient positioning, CSF drainage, venous preservation, and meticulous dissection of the deep margins are critical to this approach.

\section{References}

1. Bohnstedt BN, Kulwin CG, Shah MV, Cohen-Gadol AA: Posterior interhemispheric transfalcine transprecuneus approach for microsurgical resection of periatrial lesions: indications, technique, and outcomes. J Neurosurg 123:1045-1054, 2015

2. Chi JH, Lawton MT: Posterior interhemispheric approach: surgical technique, application to vascular lesions, and benefits of gravity retraction. Neurosurgery 59 (1 Suppl 1):ONS41-ONS49, 2006

3. Davies J, Tawk RG, Lawton MT: The contralateral transcingulate approach: operative technique and results with vascular lesions. Neurosurgery 71 (1 Suppl Operative):4-14, 2012

4. Ferroli P, Russo A, Albanese E, Tringali G, Broggi G: Gravity-aided trans-falcine removal of a contralateral subcortical ependymoma. Acta Neurochir (Wien) 149:1147-1150, 2007

5. Fronda C, Miller D, Kappus C, Bertalanffy H, Sure U: The benefit of image guidance for the contralateral interhemispheric approach to the lateral ventricle. Clin Neurol Neurosurg 110:580-586, 2008

6. Goel A: Transfalcine approach to a contralateral hemispheric tumour. Acta Neurochir (Wien) 135:210-212, 1995

7. Hafez A, Raygor KP, Lawton MT: Contralateral anterior interhemispheric approach to medial frontal arteriovenous malformations: surgical technique and results. Oper Neurosurg [epub ahead of print], 2016

8. Kim YB, Young WL, Lawton MT: Parafalcine and midline arteriovenous malformations: surgical strategy, techniques, and outcomes. J Neurosurg 114:984-993, 2011

9. Lawton MT, Golfinos JG, Spetzler RF: The contralateral transcallosal approach: experience with 32 patients. Neurosurgery 39:729-735, 1996

10. Lawton MT, Kim H, McCulloch CE, Mikhak B, Young WL: A supplementary grading scale for selecting patients with brain arteriovenous malformations for surgery. Neurosurgery 66:702-713, 2010

11. Spetzler RF, Martin NA: A proposed grading system for arteriovenous malformations. J Neurosurg 65:476-483, 1986

\section{Disclosures}

The authors report no conflict of interest concerning the materials or methods used in this study or the findings specified in this paper.

\section{Author Contributions}

Conception and design: Lawton. Acquisition of data: Burkhardt, Winkler. Analysis and interpretation of data: Lawton, Burkhardt. Drafting the article: all authors. Critically revising the article: all authors. Reviewed submitted version of manuscript: all authors. Approved the final version of the manuscript on behalf of all authors: Lawton. Statistical analysis: Burkhardt. Study supervision: Lawton.

\section{Correspondence}

Michael T. Lawton, Department of Neurological Surgery, Barrow Neurological Institute, c/o Neuroscience Publications, St. Joseph's Hospital and Medical Center, 350 West Thomas Rd., Phoenix, AZ 85013. email: michael.lawton@barrowbrainandspine.com. 